\title{
ULTRASONIC DIAGNOSIS OF URETHRAL DIVERTICULUM
}

\author{
NAOMI PLISKOW, M.D. \\ TERRY M. SILVER, M.D.
}

From the Department of Radiology, University of

Michigan Medical School, Ann Arbor, Michigan

ABSTRACT - A urethral diverticulum was diagnosed on pelvic ultrasonography. Its ultrasonic features and differential diagnosis are discussed. An awareness of the ultrasonic appearance should result in more frequent recognition of this lesion.

Urethral diverticula are common in women and may cause persistent annoying symptoms. They may go unrecognized unless the clinician has a high index of suspicion and requests specific uroradiologic or endoscopic procedures. Even then they may be difficult to identify. Ultrasonography is well suited to the noninvasive demonstration of these cystic lesions. Their recognition as unsuspected, incidental findings also may be of significant value.

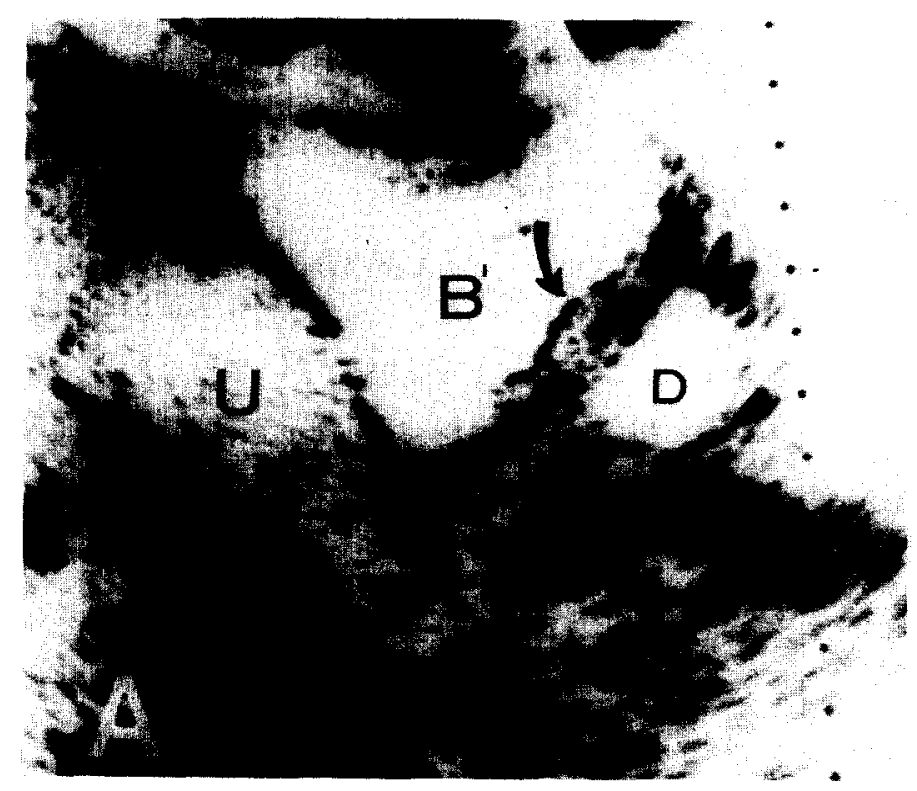

Figure 1. Sagittal (A) and transverse (B) sonograms showing urethral diverticulum posterior to urinary bladder; note echogenic "septum" between ovoid cystic mass and urinary bladder on sagittal scan (arrow). $U=$ uterus, $B^{\prime}=$ bladder, and
$D=$ urethral diverticulum.

\section{Case Report}

A thirty-onc-ycar-old white woman had a one and one-half-year history of perineal pain and dysuria. Because a mass was palpated in the left lower quadrant on physical examination, the patient was referred for a pelvic ultrasound examination. Ultrasonography showed a left adnexal cystic mass, considered to be of tubal or ovarian origin. In addition, a 2 by $3 \mathrm{~cm}$. fluidfilled structure was visualized posterior to the 


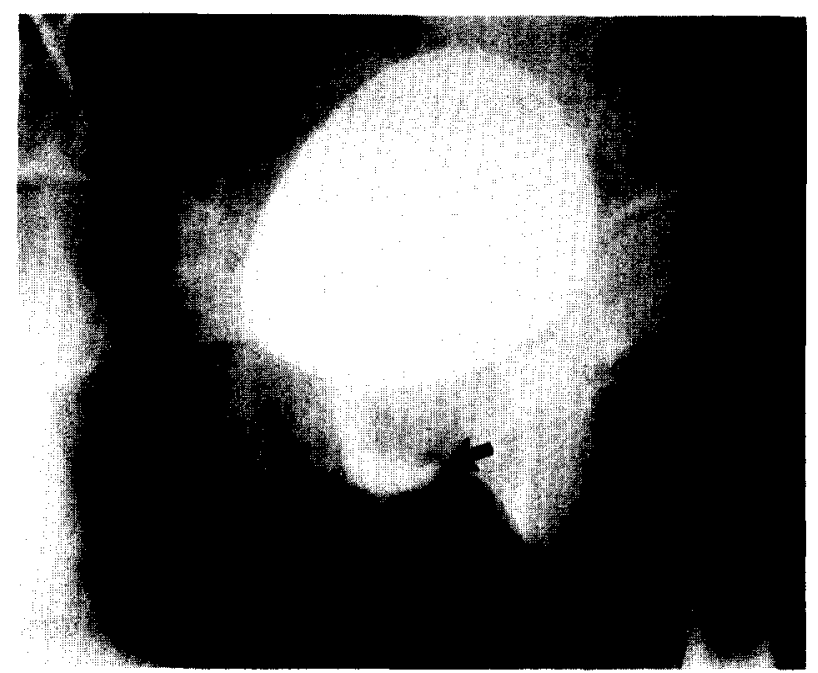

FIGURE 2. Voiding cystourethrogram: contrast opacifies urethral diverticulum (arrow) confirming ultrasonic diagnosis.

caudal margin of the bladder, in the region of the distal vagina and urethra, suggestive of a urethral diverticulum (Fig. 1). As a result of the ultrasound examination, the patient was referred to a urologist who palpated a $2 \mathrm{~cm}$., cystic mass anterior to the vagina on pelvic examination. Voiding cystourethrography confirmed the ultrasonic diagnosis of urethral diverticulum (Fig. 2). Operative excision of the diverticulum was subsequently performed with good result.

\section{Comment}

Urethral diverticula in women occur with a reported incidence of 1 to 6 per cent. ${ }^{1,7}$ They are considered to be acquired lesions secondary to obstruction of periurethral glands with subsequent infection and perforation into the urethral lumen. ${ }^{2}$ Typical symptoms of urethral diverticula in women include urinary frequency, urgency, and dysuria, ${ }^{1,3}$ although the symptoms may be ill-defined. They are diagnosed often by direct palpation, particularly if pus or urine can be extruded from the urethral orifice. Voiding cystourethrography, double balloon urethrography, or endoscopy may be required for definitive diagnosis.

Ultrasonic features of a urethral diverticulum include an ovoid cystic mass which is sharply marginated and in close proximity to the caudal posterior aspect of the urinary bladder. The ultrasonic features of our case appear to be similar to those reported by Lee and Keller in the only other ultrasonic description of this lesion.

The ultrasonic differential diagnosis of urethral diverticula in women includes fluidfilled masses of varied origin and etiology in the perivaginal or periurethral area. Gartner duct cysts may occur as large solitary masses in the midline anterior to the vaginal vault, ${ }^{4,5}$ or on the anterior or lateral aspect of the cervix or vagina. These lesions are sonographically similar to urethral diverticula. ${ }^{5}$ An endometrioma could potentially occur in this location, since a case of urethral diverticulum containing endometrial tissue on pathologic examination has becn reported. ${ }^{6}$

An ectopic ureterocele also can be visualized as a fluid-filled structure in this location, but further ultrasonic investigation usually reveals continuity with a dilated proximal ureter and upper pole hydronephrosis. Inclusion cysts are very common after surgery or trauma, but are located usually at the vaginal introitus. A urethral diverticulum can be differentiated from a bladder diverticulum by intervening echogenic tissue in the former lesion (Fig. 1A).

Since the urinary bladder is always filled for pelvic ultrasound examinations, an ideal window exists to view these lesions. Since patients with urethral diverticula often present with vague symptoms, these lesions may be difficult to diagnose clinically and sometimes radiographically. An awareness of the ultrasonic appearance should result in more frequent recognition of this lesion.

Ann Arbor, Michigan 48109

(DR. SILVER)

\section{References}

1. Davis BL, and Robinson DG: Diverticula of the female urethra: assays of 120 cases, J. Urol. 104: 850 (1970).

2. Witten DM, Myers GH, and Utz DC: Emmett's Clinical Urography, Philadelphia, W. B. Saunders Co., 1977, p. 2138.

3. Peters WA, III, and Vaughan ED: Urethral diverticulum in the female, Obstet. Gynecol. 47: 549 (1975).

4. Danforth DN: Obstetrics and Gynecology, Hagerstown, Maryland, Harper and Row, 1977, p. 894.

5. Scheible FW: Ultrasonic features of Gartner's duct cyst, J. Clin. Ultrasound 6: 377 (1978).

6. Palagiri A: Urethral diverticulum with endometriosis, Urology 11: 271 (1978).

7. Lee TG, and Keller FS: Urethral diverticulum: diagnosis by ultrasound, AJR 128: 690 (1977). 